\title{
Question de goût. L'enjeu de la modernité dans les arts et les musiques de l'Islam
}

Jean During

\section{(2) OpenEdition}

Journals

Édition électronique

URL : http://journals.openedition.org/ethnomusicologie/1314

ISSN : 2235-7688

Éditeur

ADEM - Ateliers d'ethnomusicologie

Édition imprimée

Date de publication : 31 décembre 1994

Pagination : $27-49$

ISBN : 2-8257-0503-9

ISSN : 1662-372X

\section{Référence électronique}

Jean During, "Question de goût. L'enjeu de la modernité dans les arts et les musiques de l'Islam »,

Cahiers d'ethnomusicologie [En ligne], 7| 1994, mis en ligne le 03 janvier 2012, consulté le 19 avril 2019. URL : http://journals.openedition.org/ethnomusicologie/1314

Ce document a été généré automatiquement le 19 avril 2019

Tous droits réservés 


\title{
Question de goût. L'enjeu de la modernité dans les arts et les musiques de l'Islam
}

\author{
Jean During
}

\section{NOTE DE L'ÉDITEUR}

N.B.La matière de cet article est tirée d'un chapitre de l'ouvrage du même auteur, à paraître en janvier 1995 chez Verdier, Paris : Quelque chose se passe. Le sens de la tradition dans l'Orient musical.

Il n'y a guère d'histoire que de la perception, tandis que ce dont on fait l'histoire est plutôt la matière d'un devenir, non pas d'une histoire (Deleuze et Guattari, 1980 : 428).

\section{La modernité en Occident}

1 Toute Tradition se constitue en «monde objectif » en ce qu'elle adhère à un sens, à des vérités (ou des dogmes), se conforme à des normes et des lois, est chargée de symboles qui renvoient à une transcendance faute de quoi elle déchoit en coutume. A l'opposé, la modernité peut se définir comme un vaste mouvement de "subjectivisation du monde", de «perte du monde » (Weltlosigkeit) de «désenchantement du monde» (Entzauberung) (Max Weber), c'est-à-dire l'oubli de la dimension symbolique, « le déplacement du regard du haut vers le bas » (Shayegan : 52) et le passage de la métaphysique au social.

Or, comme l'a montré Luc Ferry (1990 : 33) « l'histoire de l'Esthétique est par excellence le lieu de la subjectivisation du monde ou [...] de ce « retrait du monde » qui caractérise, au terme d'un long processus, la culture contemporaine ». Ainsi l'art occidental a cessé 
d'être le reflet du monde dans son objectivité ou sa vérité intrinsèque pour devenir un prolongement de soi. Cette mutation s'est opérée par le passage du concept de Beauté à celui de goût.

De fait, une réelle esthétique du goût - comme l'ont élaborée les Français, les Italiens et les Allemands vers le milieu du XVII ${ }^{e}$ siècle - ne peut se formuler qu'à la condition d'un retrait de la tradition métaphysique, et partant, de la Tradition en général. En effet, le goût est à la fois personnel et consensuel, mais il ne saurait prétendre à l'universalité, à l'objectivité. La Beauté n'est plus définie par rapport à la Vérité (par exemple l'ordre métaphysique ou mathématique du monde) mais est rapportée à la subjectivité : le Beau «se définit par le plaisir qu'il procure et par les sensations qu'il provoque en nous » (ibid. ):32). Au microcosme de l'œuvre d'art s'est donc substitué le monde autonome et singulier de l'individualité. Les signes concomitants de ce recul de la tradition dans les arts du Beau sont le déploiement de l'individualité, sa culmination dans le principe d'innovation et l'exigence d'originalité, lesquels définissent un nouveau type d'historicité 1 .

Dans son sens radical, en Occident, la modernité correspond donc à l'avènement du sujet et la réhabilitation du sensible, ce qui a évidemment des conséquences profondes sur les arts et la réflexion sur les arts.

5 La philosophie " classique ", se justifiant de Platon, dévalorise le monde sensible : le Beau est la forme sensible de l'Idée, la présentation sensible du Vrai. L'art porte la marque de la finitude humaine, il est en retrait du concept, en-dessous du monde intelligible. Selon Leibniz encore, le sensible n'est pour l'homme que de l'intelligible confus. Chez Hegel, l'esthétique est toujours l'expression (inférieure) du concept dans le champ de la sensibilité, bien que l'historicité de l'art soit admise. Il appartiendra à Kant de fonder, du point de vue du sujet fini, l'autonomie radicale des phénomènes par rapport à l'En-soi, l'autonomie du sensible par rapport à l'Intelligible. Inversant le mouvement traditionnel, c'est depuis le donné sensible qu'il lance sa sonde vers la métaphysique. C'est enfin Nietzsche qui, dépassant l'opposition intelligible/sensible ou modèle/copie, réhabilite le « simulacre » déprécié par Platon, et consacre l'œuvre comme son propre modèle. Ainsi le «monde-vérité » de la Tradition éclate en une multiplicité de perspectives. Le mur de la transcendance cède devant le flux impétueux de la vie, et dans cette brèche s'engouffre la modernité. Cette modernité coïncide avec l'ouverture du champ esthétique, qui suit celle de la science : «L'histoire et les sciences de la nature furent nécessaires contre le Moyen Age : le savoir contre la croyance. Contre le savoir, nous dirigeons maintenant l'art: retour à la vie! Maîtrise de l'instinct de connaissance! Renforcement des instincts moraux et esthétiques!» $(1969: 59)$

\section{Les fondements de l'esthétique islamique}

Cette modernité qui oriente les arts à partir du romantisme n'est qu'une phase d'un processus plus ancien qui fut tour à tour philosophique et épistémologique (Descartes), politique (les Lumières), idéologique et esthétique (le romantisme), avant de devenir sociologique et conquérant (le $\mathrm{XX}^{\mathrm{e}}$ siècle), et enfin post-moderne. A partir de ce constat, la question que se pose l'orientaliste est la suivante : l'Orient, dont les cultures ont atteint des sommets équivalents aux nôtres, a-t-il connu une évolution comparable, du moins en ce qui concerne les arts ? A-t-il constitué une Esthétique autonome, c'est-à-dire détachée de la métaphysique, de l'ordre cosmique, du sacré ? Ou encore, en se limitant au champ 
des cultures islamiques comme c'est la cas de cette étude, y a-t-il un concept de modernité dans les arts et plus particulièrement dans les musiques de l'Islam ${ }^{2}$, et ce concept correspond-t-il à celui qui prévaut en Occident ?

De fait, un examen attentif de l'histoire de ces cultures nous montre que, contrairement au mythe de l'Orient éternel, la pensée et les arts islamiques ont bien connu de grandes ruptures et bouleversements qui les ont conduits à une forme de modernité, ou du moins à leur propre modernité3. Sans s'étendre davantage sur ce point, l'argumentation portera ici sur le domaine de l'Esthétique, c'est-à-dire de la pensée du Beau et des arts, et en particulier de la musique. Dans un premier temps, je tenterai de définir le fond commun à la culture en question : ce qui constitue son « islamité ».

\section{La loi islamique}

8 Ce qui fait l'islamité, et donc la traditionnalité des arts islamiques, c'est la loi islamique (shar') et son observance.Au premier contact avec les sociétés islamiques on perçoit l'existence de lois et de règles auxquelles la pensée, les comportements et les productions culturelles doivent se conformer. Dans ce système, le monde est bon et beau, mais en raison de sa beauté même, il apparaît comme un piège qui détourne l'homme de la Beauté véritable et du Bien suprême. En particulier, les arts exercent un pouvoir, une sorte de magie qui menace le pouvoir religieux. Si elle n'est pas reconduite vers le divin, «la beauté paraît suspecte, sa fascination est dangereuse ou même diabolique. Au lieu d'être considérée comme le reflet du divin, elle tournera aisément en une ruse satanique " (Bürgel :7). Si les docteurs de la Loi ont contrôlé les expressions artistiques, c'est aussi dans la perspective de sauvegarder leur monde de la réduction unidimensionnelle de la mécréance, et pour contenir toute tentation de rivaliser avec Dieu, de concurrencer le paradis.

9 Toutefois, il ne faut pas se méprendre. Plutôt que des contraintes, les lois peuvent être comprises comme des précautions "écologiques" visant à établir des limites. (Par exemple, les limites à la figuration qui ne doit pas être réaliste, mais stylisée, les limites à la sensualité qui risque d'affecter la musique, etc.). La Loi peut être comprise comme définition de la mesure en toute chose, visant un équilibre: elle établit des limites qui témoignent d'une pensée cohérente, d'une vision du monde qui privilégie l'harmonie et la beauté. (Remarquons que l'existence de limites est la condition même de la création artistique. La différence est qu'en devenant autonome, l'art redéfinira dans chaque œuvre ses propres limites et règles.) Du reste, les limites sont plus ou moins imposées par un consensus de la communauté des croyants, parmi lesquels se trouvent aussi ces artistes qu'on aurait tort de considérer nécessairement comme les victimes de la Loi. Enfin, les normes islamiques sont loin d'être fixes et immuables, du moins dans les sociétés présentant un haut niveau culturel. C'est que « l'effort d'interprétation » (ijtihād) qui (on l'oublie trop souvent) est requis par le dogme islamique, fut un facteur de renouvellement et d'évolution dans les cultures qui continuèrent à l'appliquer. Ainsi la Loi peut être réinterprétée et ajustée aux exigences du temps. Les exemples sont nombreux et concernent par exemple le statut des arts figuratifs et celui de la musique.

Quant aux effets directs de la Loi sur l'art, on relèvera les points suivants :

- La rigueur de la loi a incité les artistes à concentrer leur effort créatif et, selon les mots de Nāser Farhangfar, un maître iranien contemporain, «les a poussés à creuser en profondeur ». 
- Un autre effet est que l'artiste traditionnel reste en retrait par rapport à sa création. Il ne signe pas, ne se «livre» pas lui-même dans son œuvre, dont le sujet ne peut être individualisé. Majid Kiāni, un autre maître de musique persane explique :

« Il faut conformer nos sentiments à la tradition et non pas utiliser la tradition pour notre expression personnelle. Bien sûr mon sentiment, ma pensée individuelle sont là ; ma personnalité ne disparaît pas, il ne s'agit pas de se rabaisser, de s'effacer. Mais quand je joue, j'établis un équilibre entre les sentiments. Comme le dit Hāfez,

"Ma peine est la peine de chacun"."

11 Cela favorise évidemment une esthétique du concept. Les trois grands arts sont constitués de figures typiques ou clichés : types picturaux, types mélodiques ou rythmiques (maqām, gushe, usūl), symboles et images dans la poésie. L'artiste abandonne les apparences sensibles de la nature, détourne son regard de son histoire personnelle et des manifestations sensibles de l'individualité afin de peindre des archétypes, des concepts, l'espèce universelle. On a parlé à ce propos de " point de vue de Dieu », pour exprimer le fait que l'artiste contemple le monde comme d'en haut.

Quoiqu'il en soit, la Loi n'est qu'un aspect de la culture de l'islam. Si la pensée religieuse est (ou était) sans doute normative, elle n'est pas toujours toute-puissante. L'histoire de l'art islamique est aussi celle de la transgression et de la subversion. Le système de la religion "positive", qui est grosso modo celui de la masse des musulmans, ne pouvait cependant satisfaire les intellectuels et les intuitifs. Ceux-ci ont développé leur propre système, sans renier le premier, mais en le complétant. Ce système correspond à celui de la « connaissance » ou « gnose », márifa.

\section{La gnose et la double structure du sensible}

Les docteurs de la Loi divisent le monde en ici-bas et au-delà, et l'humanité en mécréants et croyants, dont les lieux de destination sont l'enfer ou paradis. De même, ce que la pensée occidentale a retenu de la tradition, c'est l'image d'un monde à deux dimensions : physique et métaphysique, ici-bas et au-delà. Or dans ce système, la communication entre les deux mondes est impossible ou d'ordre purement spéculatif. La reconnaissance de l'autre dimension relève de la foi comme imitation, conformité à la coutume, adhésion, mais sans compréhension.

En revanche, les soufis et gnostiques ont développé une pensée multidimensionnelle et beaucoup plus complexe : leur univers se structure non pas en deux niveaux (le sensible et le supra-sensible), mais au moins en trois ou davantage. Il s'articule sur la fameuse opposition zāhir/bātin, que l'on peut traduire par le couple apparent et caché, extérieur et intérieur. Zāhir et bātin dépassent le dualisme simplifié du couple monde/outre-monde, car tous deux sont des aspects du monde. Il y a compénétration des deux niveaux, de sorte que ce couple se dédouble à nouveau en un zāhir du zāhir, un zāhir du bātin, un bātin du zāhir et un bātin du bātin. Dieu lui-même est à la fois le zāhir et le bātin, l'Apparent et le Caché, et cet Apparent est bien le lieu de la Beauté sensible.

Au lieu d'une simple séparation radicale entre le haut et le bas, le système de la gnose se caractérise par une hiérarchie du réel dans laquelle les niveaux se compénètrent, ouvrant des voies de passages. Ce monde est donc plus complexe et plus subtil, et surtout, le point important est qu'avec sa multiplicité hiérarchique de sens, il met en place la condition d'existence même de la pensée symbolique et de l'herméneutique. Cela est très important, car il n'y a pas de tradition sans herméneutique. 


\section{Mundus imaginalis}

(Kāshāni, ibid. :275) se trouvent donc toutes les formes sublimes ou terrifiantes, rangées en niveaux hiérarchiques, les plus hautes n'étant accessibles qu'à l'organe de l'imagination spirituelle, ou à l'inspiration. Car le plus important, peut-être, est que ce domaine ne s'ouvre qu'à la perception «imaginale » ou visionnaire, qui est l'organe de "l'imagination active » ou créatrice ${ }^{4}$. Nous sommes bien là dans le champ de l'art dont la spécificité est de se situer également dans une sorte d'entre-deux, qui n'est pas sans analogies avec le barzakh ${ }^{5}$, et de n'être accessible qu'à l'inspiration, à la clairvoyance, ou aux états de type extatique. De nombreux textes soufis en font foi, et le concept clef de l'esthétique musicale persane (le $h \bar{a} l$ ) ou arabe (le tarab), en est une claire référence. L'Esthétique islamique (du moins orientale) est directement liée d'une part à l'imagination visionnaire, à la vision, à la «clairaudience ", d'autre part à la perception du monde comme lieu épiphanique. Elle va donc plus loin que le monde imaginal conçu comme interface entre les idées et les formes: elle culmine dans une Esthétique de la Manifestation. «Le monde est le miroir de Dieu. La Beauté n'est jamais absente de ce miroir » (Bürgel, 1988 : 138).

ais ici apparaît une difficulté majeure pour l'élaboration d'une Esthétique autonome. Si le monde imaginal établit la possibilité d'une connexion directe entre le sensible et l'intelligible, il s'appréhende avec des organes de perception appropriés, les sens subtils (ou sens de l'âme) : œil de l'âme, oreille de l'âme. Se pose alors la question de la liaison entre ces «super-sens » qui perçoivent l'aspect «caché» (bātin) du monde, et les sens 
ordinaires qui en perçoivent l'« apparent ». En effet, les mystiques qui fréquentent les arts, en particulier la musique, en prônent souvent une perception toute intérieure. Ce n'est pas l'écoute des sons apparents qui les occupe, mais la perception de cet autre plan de réalité. Comme le dit Rūmī à propos du samā' : «tu as besoin de l'oreille du cœur, pas de celle du corps », ou « nous ne sommes pas conscients de la flûte et du tambourin »; ou encore, 'Attâr à propos de ses vers : «il faut écouter ces paroles par le coeur et l'âme ; il ne faut pas les écouter avec son soi d'eau et de terre ».

On est proche de la négation de l'art comme tel. La beauté terrestre n'est plus qu'un pâle reflet des beautés qui se dévoilent aux sens de l'âme, et l'on retombe dans le schéma classique de la Beauté comme voile de la Vérité. Si vraiment il existe une séparation radicale entre les sens "du corps » et les sens "de l'âme", on ne voit pas comment il serait possible de passer de l'un à l'autre. A moins de considérer que, de la même manière que les niveaux cosmologiques sont imbriqués, les niveaux perceptifs constituent un continuum et les facultés sont susceptibles d'affinement. Si l'art n'est pas réductible à une délectation sensible (ce que la Loi interdit), il n'est pas encore une fin en soi, car il est entièrement tourné vers sa finalité sacrée (ou sa finalité morale pour les sages et les philosophes). Quant à l'expérience esthétique, elle explose dans l'extase mystique, du moins pour la musique et la poésie. De fait, la place qui est réservée au plaisir esthétique pur (dégagé de la sensualité), mais non encore immergé dans le supra-sensible est demeurée mineure dans la pensée islamique. C'est surtout frappant pour la musique: d'un côté les adeptes de la Loi la condamnent comme diabolique parceque sensuelle, de l'autre, les mystiques la propulsent au niveau des anges et des sphères, mais sous une forme idéale, saisissable par des facultés surnaturelles. C'est entre les deux que se situe le domaine de l'Esthétique moderne au sens où elle a été développée par les penseurs occidentaux. Mais ce domaine là n'a pas donné lieu à des développements spéculatifs comparables dans la culture islamique. En bref, on écrit et on discute sur la musique soit en termes juridiques (pour la contrôler) soit en termes mystiques (pour l'exalter), soit encore en termes scientifiques et techniques, mais rarement en termes esthétiques.

\section{L'ordre cosmique et l'ordre des nombres}

La « vérité » qui constitue le «monde » de la tradition artistique islamique ne réside pas seulement au niveau du religieux ou de la métaphysique, mais aussi dans les nombres. Il convient de souligner à quel point la tradition islamique, à la suite des Grecs et peut-être des Babyloniens, a développé la part d'objectivité dans les arts du Beau par le biais des nombres, lesquels sous-tendent toute l'organisation de l'univers. L'ordre cosmique que les mystiques et les gnostiques ont projeté dans le monde des arts et des lettres fut défini avec plus de rigueur encore par les savants et les lettrés et conditionna, bien plus que la loi religieuse, les expressions artistiques islamiques.

L'exemple frappant est le développement des figures géométriques, tirées directement des nombres et des proportions. La calligraphie aussi utilise les lettres en tant que liées aux nombres par des valeurs cabalistiques. («Les secrets des lettres sont dans les nombres et les épiphanies des nombres sont dans les lettres » [Corbin, 1964 : 205]). Mais de tous les arts, c'est la musique qui reflète le mieux l'ordre de l'univers et tire son essence des structures cosmiques (sphères) et des nombres (intervalles), en d'autres termes, de l'intelligible. La musique de la période ancienne est un microcosme organisé 
qui répond à tout l'univers : le ciel avec les astres, la nature avec le chaud, le froid, les animaux, les humeurs, la physiologie, les tempéraments humains, etc.

Géométrie, calligraphie, architecture, symétrie, langage des formes, répertoires de formules, de types, rythme et mètres, intervalles, nombres, diagrammes, telles sont les grandes lignes de force de l'art islamique et aussi les conditions du classicisme, de la tradition.

Pourtant la Tradition ne tient pas ses promesses : elle est loin d'être stable, elle change constamment de visage, elle dégénère, elle est rejetée puis récupérée, ou même réinventée. Les sociétés traditionnelles aussi ont une histoire. Il nous faut voir maintenant ce qui, avec le temps, a changé dans la musique et qui peut se comprendre comme étape d'un grand processus de modernisation, ou de retrait de la Tradition. J'examinerai encore les données de la tradition musicale en signalant ce qui peut être l'indice de l'autonomisation de l'Esthétique.

\section{La finalité musicale chez les classiques et son détournement} par A. Shiloah, 1972). Sa pensée peut se résumer ainsi : la musique est à la fois une science ('ilm) et une pratique ('amal) (ibid. : 36), relevant de l'intellect ou raison ('aql) ainsi que des sentiments ou émotions (ihsās) (ibid. :42). Sa perfection réside dans l'équilibre de ces deux aspects. (Cette définition se retrouve dix siècles plus tard chez des musiciens orientaux qui pourtant n'ont pas lu les auteurs classiques). La science, donc la vérité, domine largement tous les autres aspects tels que la pratique, l'agrément des sons, ou les sentiments (ibid. : 43, 39). «La musique a pour objet d'émouvoir ceux qui possèdent sa connaissance » (ibid.: 41). On néglige donc ceux qui ont une écoute ou une pratique intuitive ou surtout émotionnelle. Plus on est connaisseur, plus on s'émeut difficilement, et inversement. Le mérite, pour le musicien, consiste non pas tant à faire les choses correctement qu'à savoir ce qu'il fait (ibid. : 180); comprendre est plus important que pratiquer (ibid. :43).

Le caractère traditionnel exemplaire de ce type de conception de l'art tient au fait qu'en privilégiant la connaissance et en dévalorisant le sensible, elle évacue totalement la spécificité du Beau. (Or, nous avons vu que les théories du Beau, en Occident, ne deviennent possibles qu'avec le repli de la métaphysique traditionnelle). Il y a cependant chez al-Kātib une amorce d'Esthétique, dans la mesure où la vérité perd du terrain devant l'art. Chez Fārābi et quelques autres, la musique est encore la fille des nombres, mais, 
chez al-Kātib, la part de vérité qui constitue la musique comme science n'est en fait qu'une connaissance entérinant des pratiques empiriques, des usages confirmés par l'habitude mais non fondés sur des lois acoustiques (mathématiques), ni non plus sur des structures cosmologiques ou métaphysiques, auxquelles il avoue ne pas croire. En ce sens, al-Kātib est un mélomane exigeant et un esthète, avant d'être un savant ou un théoricien. On pourrait dire qu'il est presque un moderne, si ce n'est que, malgré tout, il ne parvient pas à rendre compte du Beau en soi, en dehors des références à la tradition (qui doit s'entendre ici au sens mineur de coutume et norme). Il considère, comme Fārābī, que les Anciens sont parvenus à un sommet insurpassable, et perçoit la nouveauté comme une décadence. «Tout ce qui, dans la musique moderne, s'écarte des procédés de la musique ancienne, ne peut que rarement être considéré comme beau et plaire » (ibid. : 59).

\section{Le processus de décadence}

Quelle est la finalité de la musique pour les Anciens ? La valeur de la musique tient, outre à son objectivité, à son utilité et à ses vertus (al-Kātib, ibid. : 36). L'art c'est l'utile, ou éventuellement, le bien, ce qui pousse aux actions bonnes et utiles, à l'acquisition de bonnes mœurs (ibid. : 47). D'après l'auteur anonyme qui commente Fārābī (et Safioddin) quatre siècles plus tard (Sharh bar Kitāb al-adwar, trad. Erlanger, 1938), les objectifs traditionnels de la musique sont : rejoindre la poésie dans sa mission morale et spirituelle et sapientiale, et reposer l'âme de la fatigue, afin qu'elle puisse de nouveau se consacrer à des tâches utiles (recherche de la sagesse). L'auteur déplore qu'au fil des temps, les hommes aient oublié la mission morale de la musique et n'aient retenu que cette fonction de délassement et de plaisir sensible. La même chose est vraie pour la poésie :

«On ne rechercha plus que les poésies qui peuvent servir dans les jeux, et l'on ne demanda plus que les mélodies ayant le même effet que ces poésies... C'est pour toutes ces raisons que la Loi religieuse a condamné la musique. Les mélodies en faveur de nos jours appartiennent, en effet, au genre de celles que les personnes vertueuses considèrent comme viles. Jouer de la musique est ainsi devenu dégradant, parce qu'on a étendu à cet art en général le jugement porté sur le genre de musique aujourd'hui en faveur (ibid. : 549-550).

On saisit ici l'allusion à la loi islamique comme une des normes de la tradition : la musique est licite dans les limites de la moralité, tant qu'elle ne dévie pas de sa vocation éthique. Le ravissement esthétique à lui seul est suspect de déviation dans la délectation sensible.

Les critiques de Frābī se retrouvent chez al-Kātib, puis, quatre siècles plus tard, chez son commentateur. La musique n'en finirait donc pas de dégénérer. Le sens de cette décadence (ou de cette "modernisation») est qu'elle maintient l'homme dans la seule dimension sensible. Al-Kātib décrit ainsi les effets d'une telle musique : elle est sensuelle, associée aux beuveries, et ne vise que le plaisir de l'ouie (Shiloah : 44) se traduisant par une vulgaire agitation ou excitation (tahrik) (ibid.: 213). Les auditeurs la subissent passivement et s'en enivrent facilement (ibid.: 13). Son ethos (tarab), ne serait qu'une " espèce de douce délectation sensuelle ou une forte agitation s'emparant de l'auditeur non avisé à l'écoute d'une musique qui lui plaît instinctivement » (ibid. : 213).

De l'autre côté, il y a la musique sérieuse, profitable et utile, destinée au connaisseur et au savant, à «l'auditeur averti qui allie le plaisir de l'esprit et celui du cœur en interprétant les perceptions auditives au moyen de la raison " (ibid. : 13). L'émotion qu'elle suscite est un équilibre entre la raison et le coeur, avec une nette domination de la raison. Elle 
correspond à "une audition contrôlée par la raison », et procure une "délectation spirituelle, une joie intellectuelle » (ibid. : 213).

Cette opposition entre les deux types de musique, d'émotion et d'auditeurs est très ancienne et aussi très actuelle. On la trouve chez Platon (Timée 80b) et dans tous les écrits soufis sur le concert mystique samā', jusqu'au XVI ${ }^{e}$ siècle au moins. Elle est exprimée très clairement par de nombreux musiciens contemporains.

La question qui se pose alors est : s'agit-il bien là du clivage Tradition/Modernité ? Il n'y a aucun doute que pour les Anciens, il s'agit bien de cela. Il faudrait évidemment pouvoir préciser le sens que les Anciens prêtaient au concept de modernité. Cette opposition pouvait s'exprimer simplement par des contrastes formels : ce qui est bon et ce qui est mauvais. Or, il semble bien qu'elle soit rapportée à la temporalité. Dans leur système, le bon, la perfection, c'est l'ancien, car - comme en vertu d'une sélection naturelle - ce qui reste, c'est le meilleur, l'authentique, le vrai. L'ancien fut, est, et sera ; inversement ce qui sort des critères traditionnels est éphémère et ne saurait devenir ancien. C'est un effet de mode, dont on se lasse vite. Sans doute de tels genres ont aussi existé autrefois, mais ils n'ont pas subsisté. La tradition est donc synonyme de qualité, de perfection et d'équilibre. La mauvaise qualité, l'imperfection et l'absence d'équilibre sont modernes ou contemporains et ne sauraient durer, car n'est moderne que ce qui est voué à passer de mode.

«Il en est de leur nouveauté comme des fruits frais; leurs chants ne subsistent que peu de temps et périssent comme les fruits. Après quoi, ils en créent d'autres. Ils ne cessent donc d'abandonner tous les ans ce qu'ils avaient créé l'année précédente (al-Kātib, ibid. : 58)».

Ces propos coïncident parfaitement avec ceux de certains de nos musiciens contemporains orientaux. Ils les appellent également « airs du jour » en Iran ou « airs du temps » (zamānavi) en Asie Centrale, pour les opposer au Shash maqām dont on dit qu'en raison de sa perfection il est « au-dessus du temps ».

\section{Caractère des musiques modernes}

Selon al-Kātib, la musique moderne se caractérise par sa sensualité, son éclat brillant, ses effets riches et voluptueux, la pratique du jeu instrumental pour lui-même, «la variété à l'infini, le surprenant, le luxueux, bref, l'art de caractère décoratif auquel il oppose l'art ancien qui se distingue par sa simplicité, sa sobriété, sa sérénité et sa stabilité » (Shiloah : 14). Ses attaques visent en particulier les altérations apportées par certains artistes, dont le cas le plus fameux est celui du prince musicien Ibrāhim al-Mahdi (IX ${ }^{e}$ siècle). Selon le Livre des Chants, il ouvrit la querelle des Anciens et des Modernes et choqua les connaisseurs en transformant à sa guise les mélodies. En réponse il déclarait : «Je suis fils de roi, je chante comme il me plait ; et je choisis dans le ghinā' ce que je trouve agréable : car je pratique le chant en vue du tarab et non pour le profit : je chante pour moi, non pour les autres » (Kitāb al-Aghānī, cit. Jargy, 1971 : 43).

Avec la querelle des Anciens et des Modernes apparaît le caractère subjectif des critères esthétiques. On le voit notamment dans le changement de statut du texte. D'abord les thèmes antiques du désert et de la vie nomade (chez les Arabes) deviennent complètement obsolètes; la raison en est qu'ils prônent des valeurs morales de l'état tribal et témoignent d'un regard naï sur une nature encore nimbée de l'aura de l'animisme, aux antipodes des Modernes qui exaltent les thèmes spécifiquement urbains 
et sensuels de l'amour et du vin. Ensuite, le texte, c'est-à-dire ce qui assurait le sens, la vérité du chant, est rélégué au rang de prétexte pour le simple agrément de l'ouïe. A cela s'ajoute le développement de la musique instrumentale, considéré comme une bizarrerie par les Arabes. Ainsi la musique prend ses distances par rapport au verbe qui lui donnait un contenu: elle glisse dans le pur jeu des formes. De nos jours en Asie intérieure, et notamment en Iran et en Inde, la musique instrumentale a pris une importance égale à la musique vocale. Cette évolution ouvre la grande question du contenu, du sens de la musique, qui fit hésiter certains grands penseurs occidentaux, comme Kant, à reconnaître à la musique le statut d'art majeur.

Dans le domaine de la peinture également, à partir du $\mathrm{XVI}^{\mathrm{e}}$ siècle, on voit par exemple les artistes persans faire preuve d'une liberté et d'une fantaisie extraordinaires, déborder les cadres géométriques et littéraires, refuser la perspective pour se sentir plus libres (et non par souci de préserver l'essence des choses). Plus tard, l'image d'un monde bien ordonné s'estompe avec le temps, libérant la subjectivité et l'individualisme. Le thème mystique (mais parfois aussi libertin) de l'ivresse envahit la littérature, la musique et la miniature. Hāfez et ses illustrateurs menacent l'ordre cosmique et l'ordre public. Ne proclame-t-il pas : «Viens, échanson, fendons la voûte du firmament et traçons des plans nouveaux! » L'ordre du monde lui-même a-t-il été remis en question à la suite des contacts avec l'Occident? Toujours est-il qu'à la lecture des traités musicaux persans, on constate qu'à partir du XVI ${ }^{e}$ siècle la science pure fait place à des spéculations holistiques reliant entre eux tous les éléments du monde sensible et du cosmos. Et puis tout d'un coup, vers le XIX ${ }^{e}$ siècle, les correspondances symboliques n'intéressent plus les théoriciens de la musique qui se contentent de décrire, très vaguement, la pratique. Lorsque la structure musicale s'effondre en Perse, on a l'impression que c'est tout un monde, toute une vision du monde qui disparaît.

Dans la musique persane et azerbaïdjanaise, le style classique se démode et ouvre la voie à une sorte de «romantisme». Les antiques suites solidement structurées et rigides sont remplacées par un répertoire de pièces autonomes de rythme non mesuré, donc libre, affranchi des structures géométriques ou chorégraphiques. Au lieu d'un répertoire unique, chaque musicien est tenu d'interpréter le modèle (radif) à sa manière, de le paraphraser librement et de manière personnelle au cours de la performance. Cette multiplication des perspectives individuelles et cette relativisation du modèle sont à l'opposé du statut des répertoires canoniques définitivement clos qui subsistent en Asie Centrale, dans les rites soufis des Mevlevis turcs ou au Maghreb, c'est-à-dire dans des cultures qui ont préservé précisément l'ancienne tradition.

La forme musicale privilégiée est désormais le taqsim (ou le radif) qui est par essence et par définition ( $q$ sm $=$ division) un art de la discontinuité et de l'instant (puisqu'il est improvisé). Enfin le taqsim, s'il est aussi vocal (layalī) prend tout son essor dans le jeu instrumental, et conquiert progressivement son autonomie par rapport au verbe avec tout ce qu'il implique comme contraintes sémantiques, métriques et expressives. Le temps se fragmente aussi bien dans la performance libre (taqsim) que dans les pièces mesurées (« rythmées »), qui abandonnent les longs cycles (9, 12, 24, 32 temps, etc.) et les longues compositions.

43 En bref, la musique suit le même type d'évolution qui caractérise le passage du classicisme occidental au romantisme. D'ailleurs, la musique persane ou turque que l'on designe de nos jours comme traditionnelle est généralement qualifiée de "romantique ", sentimentale et subjective par ceux qui la pratiquent et l'écoutent. Le plus remarquable 
est que les idées répandues sur la musique persane correspondent aux théories esthétiques pré-romantiques, comme on va le voir.

\section{Repères pour un contrechant oriental}

biais des nombres, par la rationalité des intervalles et la mécanique de l'effet des modes. («La musique est un exercice inconscient de l'arithmétique par lequel l'esprit ne sait pas qu'il compte ", dit Leibniz.) L'idée de la structure physico-mathématique du son sera encore défendue par Rameau. L'homme vibre par sympathie avec les sons parce qu'il est également constitué de vibrations. De là le concept d'effet, qui est omniprésent dans les anciennes théories de la musique, et qui est l'équivalent exact du ta'thir arabe. A la Renaissance (peut-être sous l'influence des théoriciens de langue arabe), on se retourne vers l'Antiquité dans l'espoir de trouver chez les Grecs (ou même en Orient) les secrets du pouvoir de la musique. Ces secrets sont supposés résider dans l'alliance du verbe et des sons, ce qui par ailleurs correspond à l'exigence d'un contenu: il ne suffit pas que la mélodie soit agréable, il lui faut encore une signification. Ainsi, pour les compositeurs hyper-classiques de la Pléiade, l'intelligibilité de la musique sera assurée par le poème qui imprime son mètre, ses sonorités et sa signification selon des règles que certains croiront totalement rationnelles. On reconnaît là aussi une des préoccupations majeures des classiques orientaux et une des raisons pour laquelle ils ne prisaient guère les formes instrumentales.

Avec Rousseau, pour qui « tout chant qui ne dit rien n'est rien », l'adhésion de la musique à la langue est néanmoins d'un tout autre ordre. Liée à la langue parlante des origines, à l'expression primitive des passions, la musique devient porteuse d'un sens moral et investie de valeurs : la mélancolie originelle (chez Lacépède), la sensibilité, conduisent à l'éthique par la compassion et la pitié, et donc à l'humanité authentique. (Pour lui, celui qui prend plaisir au langage des affections ne peut être que l'homme vertueux (Chevrolet 1992: 158); il rejoint les préoccupations éthiques des maîtres traditionnels. Cette conception est également très proche de celle qui prévaut en Iran depuis probablement deux siècles, lorsque s'est opérée la rupture avec le formalisme classique, telle qu'on l'a décrite plus haut. Beaucoup d'indices donnent à penser que la musique persane elle aussi - et à la même époque - est devenue "romantique ", dans le sens où, comme chez Rousseau, elle est délivrée de l'essence mathématique, définie comme intériorité, comme langue du cœur, comme signe des passions. Elle aussi s'est dégagée des cadres rythmiques et de la symétrie, imposés en Europe par la danse, laquelle a d'ailleurs quasiment disparu au Moyen-Orient. Elle aussi, à la même époque, a su prendre ses distances par rapport au texte pour développer un genre purement instrumental, mais néanmoins "parlant». Ainsi, elle a conquis son autonomie en tant qu'activité humaine et «art du génie »résultant, comme on l'a vu, d'une vocation et d'une communication avec des plans de transcendance. Ces conceptions esthétiques peuvent paraitre banales de nos jours, mais il faut se souvenir qu'en Europe, ce n'est qu'au XIX ${ }^{\mathrm{e}}$ siècle que la musique atteint la haute signification qu'on lui prête toujours, et qu'elle commence à être prise comme pur objet de pensée, ou forme de pensée, langage se signifiant soi-même, sans qu'il ne soit plus besoin de références au sacré. Pour Kant encore - qui, sur ce point rejoint les oulémas - 
«la musique est assurément plus jouissance que culture », tandis que pour Hegel, bien qu'elle soit la langue énigmatique des passions, elle encourt l'accusation de formalisme, $\mathrm{du}$ fait de son indétermination dont seule la lumière du verbe peut la sauver. Il est vrai que c'est toujours l'avis de nombreux mélomanes orientaux, tout comme d'amateurs d'opéra ; mais ce n'est qu'avec les romantiques que la musique devient connaissance, voire révélation ${ }^{6}$.

Il est évident que les conceptions des Romantiques s'harmonisent avec celle des Orientaux, et que si Rousseau avait pu assister à leurs concerts, il y aurait trouvé l'illustration de ses idées et de ses goûts, De la même manière que Gobineau retrouvait dans l' " opéra » religieux persan (ta'zie) la tragédie grecque dans tous ses états, ou que Goethe saluait le génie de Hâfez.

Par contre, pour les Orientaux en général, l'autonomie de la musique n'est pas allée jusqu'à la négation de toute expression et l'évacuation du sujet. L'esthétique de Hanslick, culminant chez un Stravinsky avec un objectivisme musical supra-classique refusant «toute connivence signifiante avec l'auditeur »- semble toujours inadéquate pour rendre compte de l'esthétique orientale?

Avant cela, il a fallu également passer par plusieurs étapes :

- dépouiller l'art musical de ses qualités d'idole ou d'icône sonore, d'objet sacré, afin de le recentrer dans l'intériorité du sujet. C'est ce qui s'est passé en particulier dans la musique persane où depuis une cinquantaine d'année ont été exaltées les valeurs de l'inspiration, de la créativité, de l'originalité et de la personnalité, du style, de l'improvisation, au détriment de la conformité aux canons, de la fidélité aux répertoires, de la performance des compositions.

- le soustraire à sa finalité de « délectation sensible » (qui était également courante en Europe jusqu'au XVI ${ }^{\mathrm{e}}$ siècle). On assiste en effet à différentes tentatives de justifications ethiques de la musique. La plus marquée, mais aussi la plus superficielle, est l'épuration islamique qu'a connue l'Iran depuis 1980.

- renoncer aux vues mécanistes de l'effet des sons, des couleurs affectives des modes et des rythmes, en rapport avec l'ordre de la nature et du cosmos, ceci afin de rendre ses droits au « talent de l'inspiration libre » (Descartes);

- restaurer la vocation éthique et morale de la musique (Rousseau, Lacépède).

Ces quelques repères, qui mériteraient d'être développés en une véritable esthétique comparative, suffisent cependant à donner l'impression que les voies de l'Occident et de l'Orient (notamment persan) sont comme les deux parties d'un contrepoint fleuri : d'une voix à l'autre, un motif en anticipe un autre ou lui fait écho, se croisant parfois sur quelques notes ou progressant synchroniquement et parallèlement à distance consonante, sans pourtant que jamais leurs voix se confondent ou perdent leur timbre et leur registre propre.

\section{Le cadre social}

\section{Statut et attitude des artistes}

Le statut du musicien semble avoir suivi une évolution parallèle. C'est ainsi qu'en Orient se dégage la notion d'artiste qui prend le contrepied de celle d'artisan de divertissement qui définissait le musicien. 

statut sera réévalué dans la plupart des cultures islamiques à la fin du XIX et au XX siècle, probablement à l'imitation de l'Occident et avec l'avance de la laïcisation. Il y a cependant quelques anecdotes qui annoncent ce changement et montrent au que l'artiste ne veut plus se mettre au service du Prince. Sheykh Khazâne (fin XIX ${ }^{e}$ siècle) que le chah avait contraint de chanter pour lui, refusa ses présents puis, en signe de protestation et pour ne plus chanter en public, se fit arracher toutes les dents! (Caron et Safvate : 217). On trouve en Inde une anecdote également significative, représentée dans de nombreuses miniatures. On y voit, à l'inverse de la structure hiérarchique classique, l'empereur debout dans une posture modeste, tandis que son musicien de cour (Tansen) se tient à l'écoute d'un grand maître de chant, le dévot Swami Haridas qui vit à l'écart du monde (Delvoye: 225s). On dit que l'empereur s'évanouit dans l'extase causée par le chant de l'ascète, "puis demande à Tansen pourquoi il ne chante pas aussi bien que Swami Haridas; Tansen lui répond: - Je chante à la cour d'un puissant souverain, mais mon maitre chante pour Dieu». Dans une autre version il répond: "Je chante pour un souverain du monde, tandis qu'il chante pour lui-même » (ibid. : 221). On a vu que alMahdi, le prince musicien abbaside, tient des propos identiques. los mains de talentueux amateurs et de dilettantes raffinés. Le concept d'artiste prend d'art persane, et probablement aussi arabe. Je considère l'anecdote suivante comme un fait historique d'une grande signification dans l'histoire de la musique orientale : dans les années 1940, le jeune et prodigieux maître de santur Habib Somā’i se dispute avec le directeur de la radio pour protester contre la démagogie sévissant dans les programmes musicaux. Le ton monte et le maître gifle le technocrate. Cette réaction violente marque la dernière phase d'un processus que l'on peut qualifier de démocratisation ou démagogisation. Désormais la musique d'art persane est à la portée de tous, par les disques (assez onéreux au début), puis par la radio, et enfin par les cassettes dont l'essor fut bien plus rapide en Orient qu'en Occident. Tout le monde peut entendre les meilleurs artistes, et plus encore, les moins bons. Le cercle des experts est brisé, la musique déferle dans la société de consommation, chacun se croit compétent pour en juger, et le jugement de la masse consacre dorénavant les talents, envers et contre celui des experts. Voilà bien le signe de la fin de la tradition et le commencement de la modernité. Ce processus a affecté aussi la musique égyptienne : après avoir opté pour une poésie populaire et simple, les détenteurs de la grande tradition ont délibérément sacrifié l'art classique pour toucher la grande masse.

\section{Le facteur politique}

Comment en est-on arrivé là ? Y a-t-il ici un fait nouveau par rapport à la modernité à laquelle ont été confrontés les Anciens ? Et d'abord y a-t-il eu quelque facteur d'un ordre tout à fait différent qui a pu conduire à cette crise?

Peut-être convient-il de mesurer l'impact qu'ont eu sur la tradition les changements politiques survenus en Iran durant un siècle. En Europe, la modernité, c'est-à-dire le 
retrait de la Tradition et l'avènement de l'individualisme correspond à la remise en question de la légitimité et de la transmission du pouvoir, allant de pair avec la laïcisation. En ce qui concerne la tradition musicale persane, le premier recul sensible peut être repéré au début du siècle, avec l'élaboration de la Constitution et l'éveil de la démocratie. Il est vrai que plus récemment, avec la révolution islamique, l'ancien monde se restructure d'une certaine manière, mais sous les apparences de la démocratie: l'unification idéologique et l'utopie de fondre les peuples en une grande nation islamique n'ont pas réduit la diversité des formes musicales orientales et n'ont fait qu'accentuer la volonté d'autarcie des musiciens traditionnels. La musique subit de nouvelles pressions, mais (contrairement à des idées reçues) le statut de l'artiste "classique " se trouve revalorisé, du moins verbalement, en raison de son aura éthique (et dans le fond, conformément au modèle occidental). La loi islamique à nouveau appliquée ne vise pas les musiques sérieuses et "scientifiques" et ne s'attaque qu'aux formes dépravées et vulgaires (bien que ces catégories soient anciennes, l'idée de scientificité de la musique érigée en mythe - semble venir également de l'Occident, car les musiciens se soucient peu de science musicale).

\section{Valorisation de l'invention}

57 Sous la Renaissance encore, l'ancienneté, le fait d'incarner la tradition, est en soi respectable et admirable. Ce n'est plus le cas par la suite. La valeur de l'ancien, c'est l'aptitude à se conformer à une norme par essence supérieure. Or, lorsqu'il s'avère que cette norme peut évoluer, l'originalité cesse d'être une non-valeur et peut être exigée d'un artiste (Ferry : 37). Cette évolution n'est pas sans rapport avec ce qui s'est passé dans certaines musiques d'Orient, à cela près que la confrontation aux progrès et aux nouveautés occidentales a certainement modifié la perception des arts en général.

L'ancien schéma était :

stabilité de la tradition $\rightarrow$ reproduction des modèles $\rightarrow$ niveau artisanal

Le nouveau schéma devient :

idéologie du progrès $\rightarrow$ invention de nouvelles formes $\rightarrow$ niveau artistique

Ce n'est pas là la seule justification de la valorisation de l'invention. On peut penser qu'avec la perte des références transcendantes (éthiques, cosmologiques, naturelles, symboliques ou autres), un nouveau type de créativité a pu prendre son essor, une forme d'art pour l'art. Une fois la musique ramenée au domaine du pur sensible, les critères formels sont laissés au seul goût, le critère de la beauté étant l'agrément qu'elle cause, l'émotion, le tarab. Ainsi les propos d'Ibn al-Mahdi seraient pour la musique du MoyenOrient, les premiers signes d'une évolution du statut de la création et d'une volonté délibérée de rompre avec le passé. Par ailleurs, il est significatif que ces changements soient le fait du Prince, et non du simple sujet. Ses arguments annonceraient alors l'époque moderne où les maitres arabes, turcs et persans, appartenant à l'aristocratie ou à la bourgeoisie, deviennent libres de toute dépendance au public ou au mécénat et cultivent la musique pour elle-même et pour eux-mêmes. Certains voient en al-Mahdi, un précurseur arabe du romantisme. Cela reste toutefois à vérifier; peut-être ses compositions tombaient-elles dans la catégorie des chansons du jour décrites par al-Kātib.

61 Ce qui est toutefois certain, c'est qu'en tout temps (ou du moins depuis cette époque) il y eut des tensions entre deux tendances au moins que l'on peut, pour simplifier, qualifier d'innovatrice et de conservatrice. Ainsi, quelques siècles plus tard, le commentateur de 
Fārābi cité plus haut, qui dénonce par ailleurs le détournement de la finalité musicale et la perte des références traditionnelles, conspue les musiciens pour leur manque de créativité.

«De nos jours, les artistes [...] suivent aveuglément la routine, se refusant à accepter toute composition qu'ils n'ont pas déjà entendue ou qui n'a pas sa semblable dans le répertoire ancien, fût-ce une mélodie belle, élégante et agréable au plus haut degré, ou capable d'amplifier l'effet des paroles qui lui sont adaptées ; mais [comme le dit le proverbe] "on ne saurait comparer les anges aux forgerons »

(Sharh bar Kitāb al-adwār, Erlanger, 1938 : 548).»

Notons que ces critiques sont d'un tout autre ordre que celles de Fārābī. Elles ne visent pas du tout le sens de la musique, sa finalité, son système général (les intervalles et modes avec leur ethos spécifique), mais simplement la décadence du goût, de l'invention. Il s'agit là d'un trait propre à toute tradition et à toute époque : la tentation de conformisme et d'imitation servile (taqlīd).

\section{Sensuel et consensuel, une esthétique du goût}

63 Malgré les témoignages et indices cités, l'impression subsiste que la modernité en question n'est qu'une phase, un aspect d'un processus toujours attesté de décadence, inhérent à toute pratique culturelle, et qui serait la condition même de la rénovation. Le fait même que, d'une époque à l'autre, des auteurs vitupèrent contre la corruption de la musique, suggère qu'il existait bien, à côté des formes modernes, des pratiques conformes à l'esprit traditionnel. La véritable modernité aurait consisté plutôt à consacrer ces formes-là. Il reste à voir maintenant si cela n'a pas été fait d'une certaine manière.

Pour mieux mesurer la différence de perspective entre les Anciens et les Modernes, il convient d'entrer dans le cercle même de la performance musicale.

Une évaluation correcte des courants esthétiques ne doit pas s'en tenir aux écrits scientifiques ou métaphysiques (comme les grands classiques), ou aux ouvrages destinés aux corporations des musiciens. En marge du discours officiel des lettrés (sur la foi duquel on a tendance à brosser un tableau monochrome des cultures islamiques), les artistes et les amateurs avaient parfois une vision complètement indépendante des cadres métaphysiques et religieux. Soufis, artistes et mécènes s'affranchissaient de bien des aspects des lois religieuses, ou les subvertissaient. Les corporations de musiciens étaient du reste les moins religieuses de toutes, et de surcroît elles furent souvent supplantées avec le temps par des musiciens indépendants et non professionnels.

En ce qui concerne les «natures » et les « effets » intrinsèques des modes et des rythmes, les concordances entre les modes et les astres, les humeurs, les caractères psychiques, les heures du jour, les signes du zodiaque, etc., qui relèvent d'une vue hyper-traditionnelle et ordonnée du monde, il n'est pas sûr qu'on y ait attaché plus d'importance que de nos jours.

Par contre, un fin connaisseur comme al-Kâtib consacre de longues pages à décrire la pratique musicale sans s'embarasser de spéculations, dressant simplement l'inventaire prescriptif de tous les procédés procurant un plaisir esthétique. Il dit essentiellement: telle pratique est juste, telle autre mauvaise. On retrouve donc là un des traits essentiels d'une esthétique du goût qui ne veut pas s'avouer telle. Même s'il invoque les Anciens et la Raison, le fait que la musique soit une pure affaire de goût marque déjà un certain 
changement de mentalité qui permettra à d'autres d'aller plus loin et d'ouvrir à la musique le chemin d'une modernité.

La conception de la performance musicale décrite par les anciens Arabes ne préfigure-telle pas des valeurs exaltées dans l'Occident contemporain? Ainsi l'artiste dispose d'une certaine liberté et peut faire preuve de discernement et de psychologie, indépendamment de toute référence à un ordre cosmique, métaphysique ou naturel. Le public n'est pas dans une état de vénération passive devant les productions démiurgiques de l'interprète : il intervient activement dans la performance musicale par des exclamations et des remarques plus ou moins codifiées. «Pour aboutir à la perfection, une séance musicale doit reposer sur l'interaction constante entre l'interprète et son public » (Shiloah, 1994). La pratique musicale des Arabes apparaît alors comme une célébration de la beauté dans sa forme la plus sensible, sensuelle même, sans justifications philosophiques, sans les visées transcendantes des mystiques, et sans souci non plus des réprobations des docteurs de la Loi. Ce type de performance musicale créatrice, conviviale, consensuelle, communicative et interactive a été découverte seulement récemment en Occident avec le jazz et les expériences des Modernes. L'accueil qui est fait en Europe depuis quelques dizaines d'années à ce type de performance suggère assez ce qu'elle a (et avait) de "moderne ", ou de toujours actuel. Certes, d'autres peuples du monde musulman ont cédé aux excès de la ritualisation et à l'immobilisme dans la performance, mais dans l'ensemble, c'est cette ligne privilégiant l'expression individuelle des musiciens comme des auditeurs que suivirent les traditions musicales islamiques.

Il faut cependant une fois de plus nuancer ce tableau de la musique ancienne comme préfiguration de la modernité.

D'abord les artistes de l'ancien temps étaient, sauf exception, totalement dépendants de leurs patrons et d'un système de classes. Ils devaient obéir au caprice du patron et des hôtes de marque, improviser ou choisir les poèmes et les mélodies en fonction de leur attente et dans le but d'une récompense (cf. Sawa:143). (Rappelons toutefois que la situation de nos compositeurs et peintres, jusqu'à leur émancipation vers le XIX ${ }^{e}$ siècle n'était guère meilleure.)

71 Ensuite et surtout, ces pratiques musicales, pour brillantes qu'elles aient été, couraient toujours le risque de basculer dans la sensualité. Le plaisir des auditeurs n'était pas purement esthétique ou platonique. Un des ouvrages plus anciens, comme le Kitāb alAghānī « rapporte une foule d'anecdotes de la cour des Ommeyades qui font de la musique un vecteur de scandale, constamment associé à la transe, à l'ivresse et la sensualité » (Lambert, 1990 : 32). Les mélomanes se dérobaient peut-être aux cadres de la tradition islamique, mais il pesait sur eux la culpabilité vis à vis de la loi et de la morale. Le prix fort de la libération de la culture musicale était donc la déchéance dans le libertinage.

Cette sensualité qui ne se pose pas de question, soulève néanmoins celle des limites esthétiques de la musique : l'art bascule dans le pur sensible, dans la frivolité.

Pour établir dans quelle mesure on est en présence d'une réelle esthétique du goût, laquelle - comme cela s'est passé en Europe vers le milieu du XVII ${ }^{e}$ siècle - ne peut se formuler qu'avec le retrait de la tradition métaphysique, il s'agit de préciser si chez les anciens Orientaux c'était le concept de Beau (ou de Bien), ou plutôt la notion de goût ( dhawq) qui faisait autorité. Les deux termes existaient (jamāl ou husn), l'un rapporté au divin, l'autre à l'expérience individuelle, comme le hāl. Peut-être certains cercles se réclamaient-ils du premier et d'autres du second. 

tradition, si ce n'est dans ce moment de distanciation, d'interrogation, de mise en doute, de déséquilibre, mais aussi de libération qui permet de passer du niveau de l'imitation à celui de l'authenticité. Quant aux phases hautes et basses de la tradition (dont on a une illustration dans l'opposition entre samâ' soufi et concerts séculaires), il s'agirait simplement du mouvement local ou pendulaire inhérent à toute tradition, et non pas d'un fait irréversible, de portée historique.

Dans ces conditions, la modernité, en soi, ne serait pas moderne, et sa seule spécificité serait simplement qu'elle couvre un champ très large de secteurs culturels et sociaux, et qu'elle est consacrée par les intellectuels qui en font un objet de discours, voire de réflexion. La rupture avec la tradition, et la modernité elle-même, n'existeraient qu'au niveau du discours, d'une pensée, d'une critique d'art qui s'éternise sur une phase de ce processus. Il n'y aurait dès lors d'autre spécificité de la modernité que de se prendre comme objet de discours, et d'autre définition du Moderne que le fait qu'il se prétend tel. Il n'y aurait peut-être rien de nouveau, aucune modernité, si ce n'est précisément le fait de discourir sur la question.

\section{BIBLIOGRAPHIE}


BÜRGEL, Johann Christopher, 1988, The Feather of the Simurgh. The «licit magic » of the Arts in Medieval Islam. New York : New York University Press.

DELVOYE, Nalini, 1990, Tansen et la tradition des chants dhrupad en langue braj, du XVI siècle à nos jours. Thèse d'Etat pour le doctorat ès-lettres. Paris : Université de la Sorbonne nouvelle.

CARON, Nelly et SAFVATE, Dariouche, 1966, Iran. Paris : Buchet-Chastel. [Les traditions musicales].

CHEVROLET, Christine, 1992, "L'esthétique musicale de Lacépède », in H. Dufourt, J.-M. Fauquet, F. Hurard, L'esprit de la musique. Essais d'esthétique et de philosophie. Paris : Klinksieck : 151- 174.

CORBIN, Henry, 1966, Terre Céleste et Corps de Résurrection,. Paris : Buchet-Chastel. (2éd. 1979 sous le titre : Corps Spirituel et Terre Céleste).

DELEUZE, Gilles et GUATTARI, Felix, 1980, Mille plateaux. Paris : Editions de Minuit.

ELIADE, Mircea, 1969, Le Mythe de l'Eternel Retour. Paris : Gallimard.

ERLANGER, Rodolphe d', 1938, La musique arabe, vol. III. Paris : P. Geuthner.

FERRY, Luc, 1990, Homo aestheticus. L'invention du goût à l'âge démocratique. Paris : Grasset.

JARGY, Simon, 1971, La musique arabe. Paris : Que sais-je.

HEGEL, Georg Wilhelm Friedrich, 1945, Esthétique (Trad. Jankelevitch). Paris, P.U.F.

LAMBERT, Jean, 1990, La Médecine de l'âme. Musique et musiciens dans la société citadine à Sana'a. Paris : Ecole des Hautes Etudes en Sciences Sociales. [Thèse pour le doctorat en Anthropologie].

NIETZSCHE, Friederich, 1969, Le livre du philosophe. Paris.

SAWA, Dimitrie, 1989, Music Performance Practice in the early 'Abbâsid era, 123-320 AH / 750-932 AD. Toronto : Pontifical Institute of Medieval Studies.

SHAYEGAN, Dariush, 1989, Le regard mutilé. Schizophrénie culturelle : pays traditionnels face à la modernité. Paris : Albin Michel.

SHILOAH, Amnon, Al-Hasan ibn Ahmad ibn 'Alî al-Kâtib, 1972, La perfection des connaissances musicales (Kitāb Kamāl Adab al-Ghinā'). Paris : P. Geuthner. [Traduction et commentaire d'un traité de musique arabe du $\mathrm{XI}^{\mathrm{e}}$ siècle].

SHILOAH, Amnon, Al-Hasan ibn Ahmad ibn 'Alî al-Kâtib, 1994, " Notions d'esthétique dans les traités arabes sur la musique ». Cahiers des Musiques traditionnelles, 7, 51-58.

\section{NOTES}

1. «Pour le Moderne, l'homme ne saurait être créateur que dans la mesure où il est historique [...] toute création lui est interdite, sauf celle qui prend sa source dans sa propre liberté; et par conséquent tout lui est refusé, sauf la liberté de faire de l'histoire en se faisant lui-même " (Eliade, 1969: 180). Pour le Traditionnel, c'est une illusion de croire que l'homme fait l'histoire, à part quelques rares chefs dont la masse subit la loi. Selon lui, l'homme moderne n'est donc ni libre ni créateur. Au contraire, le Traditionnel, lui, « est libre d'annuler sa propre histoire par l'abolition périodique du temps » (ibid.: 181), de le transcender, de vivre dans l'éternité en un acte de renouvellement qui est éminemment créatif.

2. Le terme Islam n'est pas pris ici au sens de confession religieuse; il s'agit donc des cultures dont les musiques partagent un certain nombre de points communs et qui ont été marquées à divers titres et degrés par la culture musicale de la civilisation islamique des premiers siècles. 
Elles correspondent aux aires arabophones, iranophones, turcophones, indianophones, englobant les minorités vivant en leur sein et partageant avec celle-ci des éléments musicaux (tels que matériaux mélodiques, modes, taxinomie, ou théorie musicale, etc.).

3. Sur ce point, il s'agit souvent plus de personnalités que de grands mouvements sociaux et culturels, tels Omar Khayyām, Ibn Khaldûn, Mowlānā Rūmī ou Mollā Sadrā (pour ne citer qu'eux), ainsi que certains soufis et mystiques se situant comme en rupture avec la tradition.

4. A ne pas confondre avec « l'imagination conjecturale » qui n'est qu'une forme de vagabondage de la représentation.

5. C'est ce qu'avait entrevu Hegel: « le sensible est élevé dans l'art à l'état de pure apparence, par opposition à la réalité immédiate des objets naturels. L'œuvre artistique tient le milieu entre le sensible immédiat et la pensée pure » (Esthétique, 1945 I: 63). Remarquons que dans cet univers, la place de la musique est privilégiée par rapport aux autres arts: elle est pure forme dynamique, vibration immatérielle tout en étant bien réelle, distante à la fois de la matière et du concept.

6. Par contre, ces idées étaient bien celles des grands mystiques comme Ruzbehān ou Rūmī. Ruzbehān dit qu'en une seule séance de concert mystique, on peut parcourir les étapes de Connaissance équivalant à une vie entière. Gisuderāz affirme que l'extase provoquée par la musique peut même donner l'intelligence au soufi simple d'esprit.

7. Il existe pourtant des cas où la musique devient un pur jeu formel, logique et vide de tout contenu émotionnel, comme par exemple dans la musique instrumentale indienne, en particulier pour les percussions. Il s'agit de constructions souscrivant à une définition moderne telle que "pures formes sonores en mouvement », sans aucune autre référence qu'elles-mêmes, et, en un sens, gratuites. Toutefois, il subsiste presque toujours cette notion fondamentale de « connivence signifiante avec l'auditeur ».

8. Il est tout à fait erroné, en ce qui concerne les cultures islamiques, d'assimiler la tradition à l'imitation (taqlid), malgré certains usages que l'on fait de ce terme. Les auteurs soufis critiquent sévèrement l'imitation, et certains y voient même une forme de rupture avec la tradition religieuse.

\section{RÉSUMÉS}

L'histoire de la pensée occidentale est celle du lent repli de la tradition. La place de l'art est très importante dans la compréhension de ce processus, car comme le dit L. Ferry (:33), «la modernité se définit par un vaste processus de "subjectivisation" du monde »; or, «l'histoire de l'esthétique est par excellence le lieu de la subjectivisation du monde ou [...] de ce "retrait du monde" ". Cet article veut évaluer le chemin parcouru par la pensée et l'art islamiques au MoyenOrient et en tirer des repères permettant de déterminer s'ils en ont été tenus à l'écart ou à la lointaine arrière-garde de ce processus, ou si, au contraire, ils ont également suivi des voies qui les ont menées à un type de modernité. En l'absence d'une Esthétique constituée, on a tenté d'esquisser une théorie du beau à partir des systèmes philosophiques, des pratiques et des faits culturels. Il en ressort que l'Orient islamique a suivi une évolution analogue à celle de l'Occident, passant par une vision de Beau subordonnée à une idée de la Verité et de l'intelligible, à une sorte de modernité où l'artiste semble invité à exprimer sa subjectivité. Les parallèles sont évidents jusqu'à la période post-romantique., mais l'Orient islamique n'a jamais connu de rupture aussi radicale que celle qui a transformé les arts occidentaux, notamment la musique. En revanche, ces formes de «modernité» semblent avoir été vécues synchroniquement avec des formes 
hautement traditionnelles, si bien que l'on est amené à se demander: en quoi la modernité estelle moderne?

The history of Western thinking is one of a slow withdrawal from tradition. Art plays an important role for the comprehension of this process. Luc Ferry reminds us (?: 33): « Modernity is defined by a vast process of "subjectivising" the world »; and: " The history of aesthetics is par excellence the domain of the subjectivised world or [...] this "retreat from the world" ". This article proposes to recount the evolution of Islamic thinking and art in the Middle East in order to identify hallmarks allowing to determine whether these were relegated to the margins or the into background of this process, or whether on the contrary they also followed the pathway leading to modernity. In the absence of a constituted Aesthetics, an attempt was made to sketch a theory of beauty on the basis of philosophic systems as well as facts and practices of culture. It has appeared that the Islamic Orient underwent an evolution similar to that of the West, by embracing in passing a vision of Beauty subjected to a concept of Truth and of the intelligible, before reaching a type of modernity which seemed to beckon the artist to express his subjectivity. The parallels are manifest until the postromantic period, but the Islamic Orient never underwent a break as radical as the one which transformed the Western arts, especially in the musical field. Conversely, these forms of « modernity » seem to have been experienced at the same time as highly traditional ones, so that one may well wonder to what extent modernity is, indeed, modern.

\section{AUTEUR}

\section{JEAN DURING}

Jean During, né en 1947, est docteur ès lettres et chercheur au CNRS. Il est l'auteur de nombreuses publications sur la musique orientale. Après une formation en musique occidentale et en philosophie, il a abordé la culture iranienne. Au cours d'un séjour de neuf ans en Iran (1972-1981), il a appris la musique persane en fréquentant les grands maîtres de la tradition. Ses recherches couvrent des aspects très divers, aussi bien historiques que purement musicologiques (La musique iranienne, tradition et évolution, Paris 1984), mais il est particulièrement attaché à éclairer les relations entre la musique, la pensée traditionnelle et le sacré (Musique et mystique dans les traditions de l'Iran, Paris 1989; Musique et extase, l'audition mystique dans la tradition soufie, Paris 1988). Ses recherches l'ont poussé également vers l'Asie Centrale et l'Azerbaïdjan ( $L a$ musique traditionnelle de l'Azerbāyjan et la science des muqāms, Baden-Baden 1988). 